\title{
EDITORIAL
}

\section{INCIDENT DEMENTIA IN TRIALS OF ANTIHYPERTENSIVE TREATMENTS}

\author{
T.E. STRANDBERG ${ }^{1,2}$, A. BENETOS $^{3}$, M. PETROVIC ${ }^{4}$
}

\begin{abstract}
1. University of Helsinki, Clinicum, and Helsinki University Hospital, Helsinki, Finland; 2. University of Oulu, Center for Life Course Health Research, Oulu, Finland; 3 . Department of Geriatrics and FHU CARTAGE, CHU de Nancy and INSERM 1116, Université de Lorraine, Nancy, France; 4. Department of Geriatrics, Ghent University Hospital, and Ghent

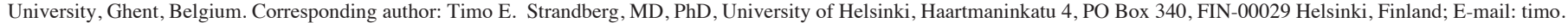
strandberg@oulu.fi; Tel: +358406724533
\end{abstract}

The Systolic Blood Pressure Intervention Trial (SPRINT) Memory and Cognition in Decreased Hypertension (MIND) study (1), failed to show a significant effect on the primary endpoint (probable dementia, hazard ratio [HR] 0.83; $95 \%$ CI, 0.67-1.04). Because this may be due to limited power, the results of SPRINT MIND were put in the context of major randomised and controlled hypertension trials (RCT) in which incident dementia had been adjudicated.

The same search strategy as in an earlier meta-analysis (up to 2015) (2) was used to identify trials which fulfilled the following criteria: 1) RCT, 2) antihypertensive treatment for at least 2 years, 3 ) dementia (not only cognitive decline or clinical impression) as an adjudicated endpoint. Trials not fulfilling those criteria were excluded. Seven trials were identified, five trials were placebo-controlled, one compared intensive vs. usual treatment (1), and in one antihypertensives were part of multidomain prevention (3). HRs with 95\% CI, and a meta-analysis of trials using random effects model and test for heterogeneity were performed with NCSS statistical software 8 (www.ncss.com).

Clinical data and HRs with 95\% CI for individual trials are shown in Table 1. In addition to total in-trial results, some subgroup and extension data, and mild cognitive impairment (MCI) for SPRINT MIND are presented. Except in the Study on Cognition and Prognosis in the Elderly (SCOPE) (small inter-group blood pressure differences), point estimates of HRs for dementia were generally below unity suggesting benefit. However, the results were statistically significant only for the Systolic Hypertension in Europe (Syst-Eur) trial extension and in SPRINT MIND when incident MCI was combined with probable dementia (1). When results of the seven trials were combined in a meta-analysis (only adjudicated dementia, $\mathrm{n}=1,297$ ), active or a more intensive antihypertensive treatment was associated with a significant reduction of dementia by $13 \%$ (95\% CI $-3 \%$ to $-23 \%, \mathrm{P}=0.011$ ). Although heterogeneity test (Cochran's $\mathrm{Q})$ was nonsignificant $(\mathrm{P}=0.60)$, the studies are admittedly very different, but this would rather give robustness to summary result. The result has also biological plausibility from observational studies (2).

To the best of our knowledge, no other intervention has similar record of dementia reduction in randomized trials as antihypertensive treatment. This message should be actively promoted in ageing societies as a feasible, usually safe, and with generic drugs also inexpensive way of dementia prevention in younger-old people. However, this may not apply to frailest and oldest patients (4).

Declaration of interests: T. Strandberg has had educational, consultative and research cooperation with several companies (including Novartis, Orion, Servier) and other entities interested in hypertension and its treatment. A. Benetos has been invited as a speaker in symposia organized by Novartis and Servier over the past 5 years. M. Petrovic reports no disclosures. The authors are members of the European Geriatric Medicine Society (EuGMS) special interest group on Cardiovascular Medicine in Older People.

\section{References}

1. SPRINT MIND Investigators for the SPRINT Research Group. Effect of intensive vs standard blood pressure control on probable dementia: A randomized clinical trial. JAMA. 2019;321:553-561 doi: 10.1001/jama.2018.21442. [Epub ahead of print]

2. Rouch L, Cestac P, Hanon O, et al. Antihypertensive drugs, prevention of cognitive decline and dementia: a systematic review of observational studies, randomized controlled trials and meta-analyses, with discussion of potential mechanisms. CNS Drugs. 2015;29:113-30

3. Moll van Charante EP, Richard E, Eurelings LS, et al. Effectiveness of a 6-year multidomain vascular care intervention to prevent dementia (preDIVA): a clusterrandomised controlled trial. Lancet. 2016;388:797-805

4. Benetos A, Petrovic M, Strandberg TE. Hypertension management in older and frail older patients Circ Res. 2019;124:1045-1060. DOI: 10.1161/ CIRCRESAHA.118.313236. 


\section{THE JOURNAL OF NUTRITION, HEALTH \& AGING@}

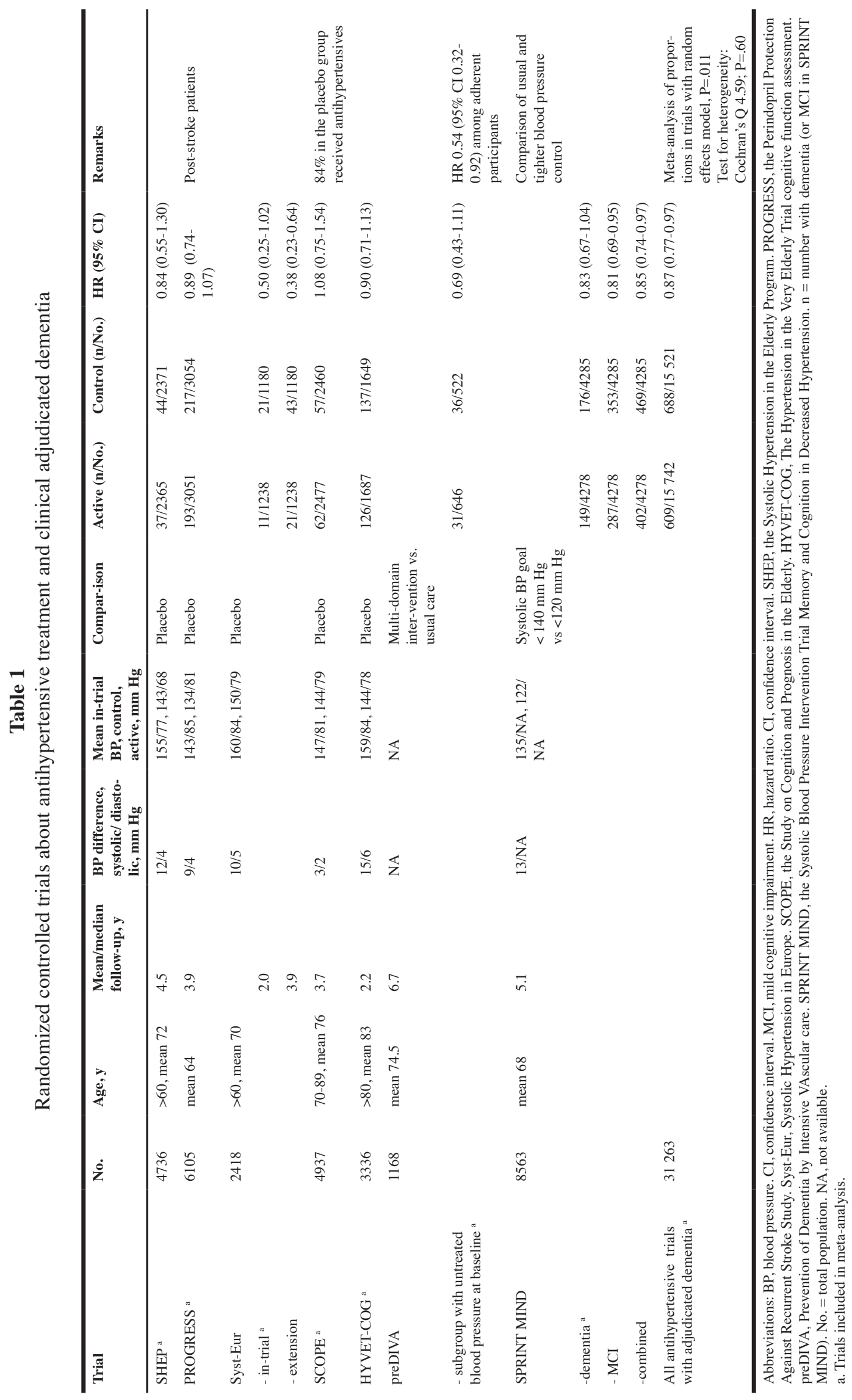

